\title{
COMPUTABLE ERROR BOUNDS FOR COLLOCATION METHODS
}

\author{
A. H. AHMED \\ Department of Mathematics \\ Ash-Sharq University \\ Khartoum, Sudan \\ (Received August 12, 1992 and in revised form February 27, 1994)
}

\begin{abstract}
This paper deals with error bounds for numerical solutions of linear ordinary differential equations by global or piecewise polynomial collocation methods which are based on consideration of the involved differential operator, related matrices and the residual. It is shown that significant improvement may be obtained if direct bounds for the error in the solution are considered. The practical implementation of the theory is illustrated by a selection of numerical examples.
\end{abstract}

KEY WORDS AND PHRASES: 1980 AMS SUBJECT CLASSIFICATION:

\section{INTRODUCTION}

This paper is concerned with computable error bounds for the solution of linear ordinary differential equations by global and piecewise polynomial collocation methods. This work is motivated by an abstract approach of error analysis such as described for example by Kantorvich and Akilov [1, Chapter XIV] and Anselone [2, Chapter I]. It extends previous work in this area by Cruickshank and Wright [3] and considers direct error bounds on the solution and its derivatives rather than via the error bounds on the highest derivatives as described in [3].

The basic idea here is to make use of the matrix involved in the numerical solution of the differential equation in computing error bounds. The relationship between various matrices and the inverse differential operator has been considered by Wright [4], Gerrard and Wright [5] and Ahmed and Wright [6]. In particular these papers are concerned with asymptotic relationships between inverse operator norms and those of matrices related to the numerical solution. The theory leads naturally to the use of matrix norms in bounding the inverse operator norms. Then computation of the residual norm leads immediately to computation of the error bounds.

The use of these ideas in error estimation is discussed in [7]. Their practical implementation in selection criteria for adaptive collocation algorithms is examined in [8]. References to alternative approaches in collocation algorithms and error analysis such as Delves [9], De Boor and Swartz [10], De Boor [11] and Russell and Christiansen [12] may be found in [7]. Detailed comparisons with the algorithms of Ascher, Christiansen and Russell (used in the well known code COLSYS for solving boundary value problems) [13], is presented in [8].

Throughout this paper the analysis and illustrative results use infinity norms, though some of the ideas could be extended to other norms. The assumptions about the methods and equations are introduced when needed, to emphasize their significance. The theoretical background is presented only briefly as it is reasonably well known. The results are tested on a selection of problems and comparisons are made with those in [3]. The work presented in this paper is based on Ahmed thesis [14]. 


\section{ASSUMPTIONS AND NOTATION}

In order to be able to treat both global and piecewise polynomial collocation in a uniform manner we follow very closely the assumptions and notations used in [7]. We consider the linear $m$ th order differential equation of the form:

$$
x^{(m)}(t)+\sum_{j=0}^{m-1} P,(t) x^{(\omega)}(t)=y(t)
$$

With $m$ associated homogeneous boundary conditions. Without loss of generality we assume that the equation holds in $[-1,1]$. The equation may be written in operator form:

$$
\left(D^{m}-T\right) x=y
$$

where $D$ denotes the differential operator. In (2) we suppose that $x \in X$ and $y \in Y$ where $X$ and $Y$ are suitable Banach spaces. The operators $\left(D^{m}-T\right)$ and $D^{m}$ with the associated conditions are both assumed to be invertible. The approximate collocation solution is taken in a subspace $X_{n q} \subset X$. To define this, we first define a subspace $Y_{n q} \subset Y$. Suppose the interval $[-1,1]$ is subdivided by the break points $-1=t_{0}<t_{1}<\ldots<t_{n}=1$. In each sub-interval $q$ collocation points are used chosen as:

$$
\xi_{j k}=\left(\left(t_{k}-t_{k-1}\right) \xi_{j}^{*}+\left(t_{k}+t_{k-1}\right)\right) / 2 \begin{array}{r}
j=1, \ldots, q \\
k=1, \ldots, n
\end{array}
$$

where $\left\{\xi^{*}\right\}, j=1, \ldots, q$, are given reference points in $(-1,1)$. The space $Y_{n q}$ consists of functions which are polynomials of degree $q-1$ in each of the intervals $J_{k}=\left[t_{k-1}+, t_{k}-1\right], k=1, \ldots, n$. No assumptions regarding continuity at the break points is made. The solution space $X_{n q}$ is then taken as $\left(D^{m}\right)^{-1} Y$. The projection operator $\phi_{n q}$ is defined as the operator which gives the interpolant in $X_{n q}$ based on the collocation points $\left\{\xi_{j k}\right\}$. With these assumptions the approximate solution $x_{n q}$ satisfies:

$$
\left(D^{m}-\phi_{n q} T\right) x_{n q}=\phi_{n q} y \text {. }
$$

In [6] certain matrices $Q$ were introduced and their properties examined. Here we use a special case of this and denote it by $Q_{n q}$. This is most conveniently defined by considering the right hand side $y$ and the solution at the collocation points. Then there is a matrix $Q_{n q}$ such that

$$
\mathrm{x}^{(0)}=Q_{n q} \mathbf{y}
$$

and this can be regarded as a definition of $Q_{n q}$. In a similar way we define the matrix $Q_{n q}^{(r)}$ by

$$
\mathbf{x}^{(r)}=Q_{n q}^{(r)} \mathbf{y}, \quad r=1,2, \ldots, m-1
$$

and the matrix $Q_{n q}^{(m)}$ by

$$
\mathbf{x}^{(m)}=Q_{n q}^{m} \mathbf{y}
$$

Then under suitable conditions it was shown in [6] for $r=0,1, \ldots, m-1$ that

$$
\begin{gathered}
\left\|Q_{n q}^{(r)}\right\| \rightarrow\left\|D^{r}\left(D^{m}-T\right)^{-1}\right\| \text { as } n \rightarrow \infty, q \text { fixed, and } \\
\left\|Q_{n q}^{(r)}\right\| \rightarrow\left\|D^{r}\left(D^{m}-T\right)^{-1}\right\| \text { as } n \rightarrow \infty, n \text { fixed, and }
\end{gathered}
$$

in [4] and [5] that

$$
\begin{gathered}
\left\|Q_{n q}^{(m)}\right\| \rightarrow\left\|\left(I-T\left(D^{m}\right)^{-1}\right)\right\| \quad \text { as } n \rightarrow \infty, q \text { fixed, and } \\
\left\|Q_{n q}^{(m)}\right\| \rightarrow\left\|\left(I-T\left(D^{m}\right)^{-1}\right)\right\| \quad \text { as } n \rightarrow \infty, n \text { fixed }
\end{gathered}
$$


These conditions concerned the location of the collocation points and required the continuity of the coefficients $P,(t)$ in (1). In particular, the global case $(q \rightarrow \infty, n=1)$ assumed that the points $\left\{\xi^{*}\right\}$ were zero of certain orthogonal polynomials.

Some further definitions and notations are needed before constructing the bounds. It is convenient here to define the compact operators $K$ and $K_{1}$ by

$$
\begin{array}{ll}
K=T\left(D^{m}\right)^{-1} & \\
K_{1}=D^{r-m} \quad \text { for } r=1,2, \ldots, m-1 .
\end{array}
$$

We also introduce the evaluation operator $\Phi_{n q}: Y \rightarrow R^{n q}$ to give a vector consisting of the values of a function at the collocation points. Further an extension operator $\Psi: R^{n q} \rightarrow Y$ can be defined to give a function whose values at the collocation points agree with the components of the vector, with $y$ the properties $\left\|\Phi_{n q}\right\|=\left\|\Psi_{n q}\right\|=1, \Phi_{n q} \phi_{n q}=\Phi_{n q}$ and $\Phi_{n q} \Psi_{n q}=I_{n q}, \phi_{n q}=\phi_{n q} \Psi_{n q} \Phi_{n q}$, as described in [4,5]. Operator expressions for the matrices $Q_{n q}$ can now be obtained. From (4)

$$
x_{n q}=\left(D^{m}-\phi_{n q} T\right)^{-1} \phi_{n} y
$$

and applying $\Phi_{n q}$ gives

$$
\Phi_{n q} x_{n q}=\Phi_{n q}\left(D^{m}-\phi_{n q} T\right)^{-1} \phi_{n q} y
$$

and hence

$$
Q_{n q}=\Phi_{n q}\left(D^{m}-\phi_{n q} T\right)^{-1} \phi_{n q} \psi_{n q}
$$

In a similar way we express $Q_{n q}^{r}$ and $W_{n q}$ in the following operator forms

$$
Q_{n q}^{(r)}=\Phi_{n q} D^{r}\left(D^{m}-\phi_{n q} T\right)^{-1} \phi_{n q} \psi_{n q}
$$

and

$$
W_{n q}=Q_{n q}^{(m)}=\Phi_{n q}\left(I-\phi_{n q} K\right)^{-1} \phi_{n q} \psi_{n q} \text {. }
$$

\section{THE ERROR BOUNDS}

Suppose an approximate solution $x_{n q}$ of the differential equation (2) has been found and $x_{n q}$ satisfies (4). Let the residual $r_{n q}$ be defined by

$$
r_{n q}=\left(D^{m}-T\right) x_{n q}-y
$$

and the error by

$$
e_{n q}=x_{n q}-x
$$

Using (2), we have

$$
\left(D^{m}-T\right) e_{n q}=r_{n q} \text { or } e_{n q}=\left(D^{m}-T\right)^{-1} r_{n q} .
$$

It immediately follows that

$$
\left\|e_{n q}\right\| \leq\left\|\left(D^{m}-T\right)^{-1}\right\|\left\|r_{n q}\right\|
$$

Note that $\left\|r_{n q}\right\|$ can be evaluated at any point without difficulty since $x_{n q}$ is a piecewise polynomial. Thus $\left\|r_{n q}\right\|$ may be estimated by evaluation at a suitably fine grid of points. Now once a bound on $\left\|\left(D^{m}-T\right)^{-1}\right\|$ has been obtained, (15) can be used to bound $\left\|e_{n q}\right\|$. To bound $\left\|\left(D^{m}-T\right)^{-1}\right\|$ directly we need to extend the theory in [3] in the following way. If we denote the inverses $\left(D^{m}-\phi_{n q} T\right)^{-1}$ and $\left(I-\phi_{n q} K\right)^{-1}$ when restricted to the subspace $Y_{n q}$ by $\left(D^{m}-\phi_{n q} T\right)^{-1} Y_{n q}$ and $\left(I-\phi_{n q} K\right)^{-1} Y_{n q}$ then the following relation can be seen between them. 


$$
\begin{gathered}
\left(I-\phi_{n q} K\right)^{-1}=I+\left(I-\phi_{n q} K\right)^{-1} Y_{n q} \phi_{n q} K \\
\left(D^{m}-\phi_{n q} T\right)^{-1}=D^{m}+\left(D^{m}-\phi_{n q} T\right)^{-1} Y_{n q} \phi_{n q} K
\end{gathered}
$$

which implies

$$
\begin{gathered}
\left\|\left(I-\phi_{n q} K\right)^{-1}\right\| \leq 1+\left\|\left(I-\phi_{n q} K\right)^{-1} Y_{n q}\right\|\left\|\phi_{n q} K\right\| \\
\left\|\left(D^{m}-\phi_{n q} T\right)^{-1}\right\| \leq\left\|\left(D^{m}\right)^{-1}\right\|+\left\|\left(D^{m}-\phi_{n q} T\right)^{-1} Y_{n q}\right\|\left\|\phi_{n q} K\right\|
\end{gathered}
$$

Now $\left(D^{m}-T\right)^{-1}$ can be related to $\left(I-\phi_{n q} K\right)^{-1}$ and $\left(D^{m}-\phi_{n q} T\right)^{-1}$. By applying Anselone [2, page 59] to the identity

$$
\left.\left.\left(D^{m}-t\right)\left(D^{m}\right)^{-1}\right)\left(I+K+\ldots+K^{d-1}+\left(I-\phi_{n q} K\right)^{-1}\right) K^{d}\right)=I+\left(\phi_{n q}-I\right) K\left(I+\phi_{n q} K\right)^{-1} K^{d}
$$

we conclude that

$$
\left\|\left(D^{m}-T\right)^{-1}\right\| \leq\left\|\left(D^{m}\right)^{-1}\right\|+\frac{\left\|\left(D^{m}\right)^{-1}\right\|\|K\|+\ldots+\left\|\left(D^{m}\right)^{-1}\right\|\|K\|^{d-1}\left\|\left(D^{m}-\phi_{n} T\right)^{-1}\right\|\left\|K^{d}\right\|}{1-\delta_{n}^{d}}
$$

if

$$
\delta_{n}^{d}=\left\|\left(\phi_{n q}-I\right) K\left(I-\phi_{n q} K\right)^{-1} K^{d}\right\|<1 \quad d=1,2, \ldots
$$

Now to apply the extended projection theory suggested in [3], define $W_{n q} z$ by

$$
W_{n q} z=\left(D^{m}\right)^{-1} y+\left(D^{m}\right)^{-1} T x_{n q}
$$

By definition of $\phi_{n q}\left(D^{m}\right)^{-1} \phi_{n q} D^{m}$ is a bounded linear projection from $X$ to $X_{n q}$. Define $T_{n q}: X \rightarrow Y$ by $T_{n q}=T\left(D^{m}\right)^{-1} \phi_{n q} D^{m}$. Then it can easily be verified that,

$$
\begin{aligned}
& \left(D^{m}-T_{n q}\right) W_{n q}=y, \\
& \left(D^{m}-T_{n q}\right)^{-1}=\left(D^{m}\right)^{-1}+\left(D^{m}\right)^{-1} T\left(I-\phi_{n q} T\right)^{-1},
\end{aligned}
$$

and

$$
\left(K-K_{n q}\right)^{-1}=I+K\left(I-\phi_{n q} K\right) y_{n q} .
$$

Now by applying Anselone [2, page 59] to the identity

$$
\left(D^{m}-T\right)^{-1}=\left(D^{m}\right)^{-1}\left(I+K+\ldots+K^{d-1}+\left(I-K \phi_{n q}\right)^{-1} K^{d}\right)\left(I+K\left(\phi_{n q}-1\right)\left(I-K \phi_{n q}\right)^{-1} K^{d-1}\right)
$$

we conclude that

$$
\left\|\left(D^{m}-T\right)^{-1}\right\| \leq \frac{\left\|\left(D^{m}\right)^{-1}\right\|+\left\|\left(D^{m}\right)^{-1}\right\|\|K\|+\ldots+\left\|\left(D^{m}\right)^{-1}\right\|\left\|K^{d-1}\right\|+\left\|\left(D^{m}-T_{n}\right)^{-1}\right\|\left\|K^{d}\right\|}{1-\Delta_{n}^{d}}
$$

if

$$
\Delta_{n}^{d}=\left\|\left(\phi_{n q}-I\right) K\left(I-\phi_{n q} K\right)^{-1} K^{d}\right\|<1 \quad d=1,2, \ldots
$$

Now if we recall equation (9)

$$
Q_{n q}=\Phi_{n q}\left(D^{m}-\phi_{n q} T\right)^{-1} \phi_{n q} \psi_{n q},
$$

and use $\phi_{n q} \Psi_{n q} \Phi_{n q}=\phi_{n q}, \phi_{n q} x_{n q}=x_{n q}, \Phi_{n q} \psi_{n q}=I_{n q}$ and $\Phi_{n q} \phi_{n q}=\Phi_{n q}$ we get

$$
\phi_{n q} \Psi_{n q} Q_{n q} \Psi_{n q}=\phi_{n q} \Psi_{n q} \Phi_{n q}\left(D^{m}-\phi_{n q} T\right)^{-1} \phi_{n q} \Psi_{n q} \Phi_{n q}=\left(D^{m}-\phi_{n q} T\right)^{-1} Y_{n q}
$$

so that

$$
\left\|\left(D^{m}-\phi_{n q} T\right)^{-1} Y_{n q}\right\| \leq\left\|\phi_{n q}\right\|\left\|Q_{n q}\right\|
$$


If we recall (11) and use the same idea we get $\left(D^{m}-T\right)^{-1}<$

$$
\left\|\left(D^{m}-\phi_{n q} T\right)^{-1} Y_{n q}\right\| s\left\|\left(D^{m}\right)^{-1} \phi_{n q}\right\|\left\|Q_{n q}^{m}\right\| .
$$

Again, if we recall (21) and use the same idea of (23), we get

$$
\left\|\left(D^{m}-T_{n q}\right)^{-1}\right\| \leq\left\|\left(D^{m}\right)^{-1} T \phi_{n q}\right\|\left\|Q_{n}\right\|+\left\|\left(D^{m}\right)^{-1}\right\| .
$$

In a similar way it can be shown that $K\left(I-Q_{n q} K\right)^{-1}=K \phi_{n q} \Psi_{n q} Q_{n q}^{m} \Phi_{n q}$ and hence

$$
\left\|\left(\phi_{n q}-I\right) K\left(I-\phi_{n q} K\right)^{-1} K^{d}\right\| \leq\left\|\left(\phi_{n q}-I\right) K^{d+1}\right\|+\left\|\left(\phi_{n q}-I\right) K \phi_{n q}\right\|\left\|Q_{n q}^{m}\right\|\left\|K^{d+1}\right\|
$$

and

$$
\left\|K\left(\phi_{n q}-I\right)\left(I-K \phi_{n q}\right)^{-1} K^{d}\right\| \leq\|K\|\left\|\left(\phi_{n q}-I\right) K^{d}\right\|+\|K\|\left\|\left(\phi_{n q}-I\right) K \phi_{n q}\right\|\left\|Q_{n q}^{m}\right\|\left\|K^{d}\right\| .
$$

Now if we denote the bounds in (20) by $Q P_{d}$ and use (18), (19), (23), (24) and (26) we get $\left\|\left(D^{m}-T\right)^{-1}\right\|<$

$$
Q P_{d}=\left\|\left(D^{m}\right)^{-1}\right\| \sum_{i=0}^{d} \frac{\left\|K^{i}\right\|+\left\|\phi_{n q}\right\|\left\|Q_{n q}\right\|\left\|\phi_{n q} K^{d+1}\right\|}{1-\delta_{n}^{d}}
$$

and

$$
W P_{d}=\left\|\left(D^{m}\right)^{-1}\right\| \sum_{i=0}^{d} \frac{\left\|K^{i}\right\|+\left\|\left(D^{m}\right)^{-1} \phi_{n q}\right\|\left\|Q_{n}^{m}\right\|\left\|\phi_{n q} K^{d+1}\right\|}{1-\delta_{n}^{d}}
$$

If

$$
\delta_{n}^{d}=\left\|\left(\phi_{n q}-I\right) K^{d+1}\right\|+\left\|\left(\phi_{n q}-I\right) K \phi_{n q}\right\|\left\|Q_{n q}^{m}\right\|\left\|K^{d+1}\right\| \leq 1 \quad d=0,1,2, \ldots
$$

and if we denote the bounds in (22) by $Q A_{d}$ and use (21), (25), and (27) we get $\left\|\left(D^{m}-T\right)^{-1}\right\|<$

$$
Q A_{d}=\|\left(D^{m}\right)^{-1} \sum_{i=0}^{d} \frac{\left\|K^{i}\right\|+\left\|\left(D^{m}\right)^{-1} T \phi_{n q}\right\|\left\|Q_{n q}\right\|\left\|K^{d}\right\|}{1-\Delta_{n}^{d}} .
$$

If

$$
\Delta_{n}^{d}=\|K\|\left\|\left(\phi_{n q}-I\right) K^{d}\right\|+\|K\|\left\|\left(\phi_{n q}-I\right) K \phi_{n q}\right\|\left\|Q_{n q}^{m}\right\|\left\|K^{d}\right\| \leq 1 \quad d=1,2, \ldots .
$$

Following the arguments of (28), (29) and (30) and using (10) instead of (9) similar bounds for the derivatives of the solution can be derived.

Now in order to compute these bounds we need to compute bounds for $\left\|\left(\phi_{n q}-I\right) K^{d}\right\|,\left\|\phi_{n q} K^{d}\right\|$, $\left\|\left(\phi_{n q}-I\right) K \phi_{n q}\right\|,\left\|D^{m-1} \phi_{n q}\right\|,\left\|D^{r-m} \phi_{n q}\right\|,\left\|D^{m-1} T \phi_{n q}\right\|,\left\|D^{r-m} T_{n q}\right\|,\|K\|,\left\|D^{m-1}\right\|,\left\|D^{r-m}\right\|, D^{m-1} T$ and $D^{r-m} T$. Bounds for the projected terms can be computed using Jackson theorem [15] whereas bounds for the constant terms can be evaluated directly as described in [3].

\section{NUMERICAL RESULTS}

We present here a selection of numerical examples illustrating how the ideas can be applied in practice and what sort of results can be obtained. We use zeros of Tchebychev polynomials as collocation points and for the piecewise case we work with two points only. For comparison we include the bounds derived in [3] and denote them by $P_{d}$ and $A_{d}$. We also use the problems used there as basic test problems. Other problems have been considered, including higher order equations. 
Problem 1

Problem 2

$\underline{\text { Problem } 3}$

Problem 4

$$
\begin{array}{ll}
x^{\prime \prime}+\alpha\left(1+t^{2}\right) x=y & x( \pm 1)=0 \\
x^{\prime \prime}-\alpha x=y & x( \pm 1)=0 \\
x^{\prime \prime}-\frac{2 \alpha}{(t+5)^{2}} x=y & x( \pm 1)=0
\end{array}
$$$$
x^{\prime \prime}+\frac{2 \alpha x^{\prime \prime}}{t+3}-\frac{2 \alpha x}{(t+3)^{2}}=y \quad x( \pm 1)=0
$$

The parameter $\alpha$ is included to vary the stiffness of the problem. In table 1 we present the values of $P_{d}, W P_{d}, Q P_{d}, A_{d}$ and $Q A_{d}$ for the global collocation method. In comparing these bounds we observe the following inequalities:

$$
\begin{array}{lll}
\text { Problem 1 } & \alpha=.5 & A_{1}<Q A_{1} \ll P_{Q}<W P_{0}<Q P_{0} \\
\text { Problem 2 } & \alpha=.5,1 & A_{2}<Q A_{2}<W P_{1}<Q P_{1}<P_{1} \\
& \alpha=.5 & A_{1}<Q A_{1} \ll P_{0}<W P_{0}<Q P_{0} \\
& \alpha=1 & Q A_{1}<A_{1} \ll Q P_{0}<W P_{0}<P_{0} \\
\text { Problem 3 } & \alpha=.5,1 & Q A_{2}<Q P_{1}<A_{2}<W P_{1}<P_{1} \\
& \alpha=.5,1 & Q A_{1}<A_{1}<P_{0}<W P_{0}<Q P_{0} \\
\text { Problem 4 } & \alpha=.5,1 & Q A_{2}<A_{2}<P_{1}<W P_{1}<Q P_{1} \\
& \alpha=.5 & Q A_{1}<A_{1} \\
& \alpha=.5 & Q A_{2}<A_{2}<Q P_{1}<W P_{1}<P_{1}
\end{array}
$$

From these inequalities and the results in the tables we note:

(1) The extended projection bounds $\left(Q A_{1,2}, A_{1,2}\right)$ are much more accurate than the projection method bounds $\left(Q P_{0,1} P_{0,1}\right)$. This confirms expectation based on the expressions for these bounds as $Q P_{d}$ and $P_{d}$ include the projection norm $\left\|\phi_{n q}\right\|$ which is not uniformly bounded.

(2) If we consider each projection method separately, we observe that (with the extended projection method) in all problems except problem 1, $Q A_{1,2}<A_{1,2}$. For the usual projection method in most cases $Q P_{0}$ and $W P_{1}$ have no improvements over $P_{0}$, while $Q P_{1}$ and $W P_{1}$ are better than $P_{1}$. In general, if $\|K\|<1 / 2$, it can be seen that $W P_{d}<P_{d}$ and superiority of bounds using the matrix $Q$ becomes more clear when $\left\|Q_{n q}\right\| \ll\left\|\left(D^{m^{-1}}\right)\right\|\left\|W_{n q}\right\|$ as justified by the theory.

(3) If we compare bounds with small values of $d$ with those of higher values of $d$ we observe that the latter are always better. This is obviously due to the bounds derived for $\left\|\left(I-\phi_{n q}\right) K^{d}\right\|$ as mentioned in [3].

Results for the piecewise collocation method are presented in table 2. All the problems there have consistently satisfied the inequality

$$
Q A_{1,2}<Q P_{0,1}<W P_{0,1}<A_{1,2}<P_{0,1} .
$$

This confirms the superiority of bounds using the matrix $Q$ and the improvement of $P_{d}$ by $W P_{d}$ as expected by the theory.

NOTE: The missing values in the tables are due to the deltas not being $<1$. 


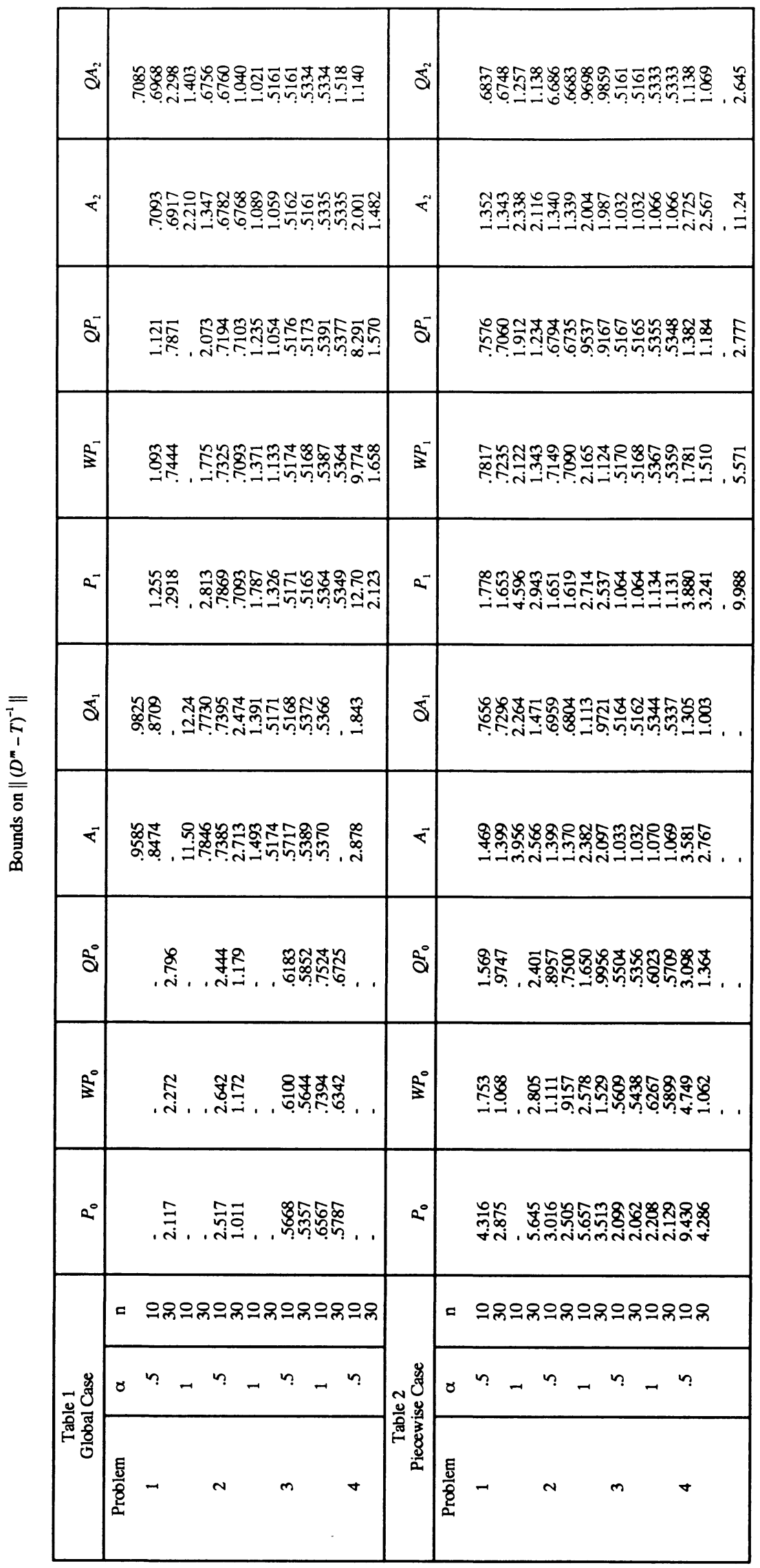




\section{CONCLUSION}

The numerical results show that improved computable bounds for the inverse of the differential operator can indeed be found if we consider the differential equation in the original form,

$$
\begin{aligned}
& \left(D^{m}-T\right) x=y, \quad \text { instead of the transformed one } \\
& (I-K) x=y .
\end{aligned}
$$

The introduction of the matrix $Q_{n q}$ whose norm as shown in [6], tends to the norm of the inverse differential operator is a main factor of the closeness of these bounds. The improvement is more obvious with the piecewise collocation method than with the global case. Within the global case there is much improvement with the extended projection method. This is clearly due to the involvement of the projection norm (which is not uniformly bounded) in the latter case. However in that case we have got $W P_{d}$ bounds which use $\left\|\left(D^{m^{-1}}\right) \phi_{n q}\right\|\left\|W_{n q}\right\|$, instead of $\left\|\phi_{n q}\right\|\left\|Q_{n q}\right\|$, as alternatives. Despite the closeness of these bounds, unfortunately the conditions of applicability $\left(\delta_{n}^{d}<1, \Delta_{n}^{d}<1\right)$, which were major problems with the previous analysis [3], have turned out to be the same. An alternative approach to deal with such problems is to split the differential operator in some different way. For example $\left(D^{m}\right)$ in (2) may be replaced by $a_{m} D^{m}+a_{m-1} D^{m-1}+\ldots+a_{1} D$ where $a_{1}, a_{2}, \ldots, a_{m}$ are some constants to be chosen to give the highest possible applicability. This idea is justified by the result described in [16] and may be investigated in a separate paper.

\section{REFERENCES}

[1] KANTORIVICH, L. V. and AKILOV, G. P., Functional Analysis in Normed Spaces, Pergamon Press, New York (1964).

[2] ANSELONE, P. M., Collectively Compact Operator Approximation Theory, Prentice-Hall, Englewood Cliffs, NJ (1971).

[3] CRUICKSHANK, D. M. and WRIGHT, K., Computable error bounds for polynomial collocation methods, SIAM J. Num. Anal, 15, 134-151 (1978).

[4] WRIGHT, K., Asymptotic properties of collocation matrix norms 1: global polynomial approximation, L.A.A.J. Num. Anal. 4, 185-200 (1984).

[5] GERRARD, C. and WRIGHT, K., Asymptotic properties of collocation matrix norms 2: piecewise polynomial approximation, I.M.A.J. Num. Anal, 4, 363-373 (1984).

[6] AHMED, A. and WRIGHT, K., Further asymptotic properties of collocation matrix norms, I.M.A.J. Num. Anal. 5, 235-241 (1985).

[7] AHMED, A. and WRIGHT, K., Error estimation for collocation solution of linear ordinary differential equations, Comp. \& Maths, with Appls., Vol. 128, No. 5/6, 1053-1059 (1986).

[8] WRIGHT, K., AHMED, A. H. and SELEMAN, A. H., Criteria for mesh selection in collocation algorithms for ordinary differential boundary value problems, L.M.A.J. Num. Anal. 11, 7-20 (1991).

[9] DELVES, L. M., in Modern Numerical Methods for Ordinary Differential Equations (Edited by G. Hall and J. M. Watt), Chap. 19, Oxford University Press (1976).

[10] DE BOOR, C. and B. SWARTZ, Collocation at Gauss points, SIAM J. Num. Anal, 10, 582-606 (1973).

[11] DE BOOR C., Good approximation by splines with variable knots II. In proceeding of a conference on the Numerical Solution of Differential Equations (Edited by G. A. Watson), lecture notes in Matheamtics 363, Springer, Berlin (1974).

[12] RUSSELL, R. D. and CHRISTIANSEN, J., Adaptive mesh selection strategies for solving boundary value problems, SIAM J. Num. Anal, 15, 59-80 (1978).

[13] U. ASCHER, CHRISTIANSEN, J. and RUSSELL, R. D., COLSYS - A Collocation Code for Boundary-Value Problems, Codes for Boundary Value Problems in Ordinary Differential Equations, Ed. B. Childs et al., New York, Springer Verlag, 164-185 (1979).

[14] AHMED, A., Collocation alogrithms and error analysis for approximate solutions of ordinary differential equations, Ph.D. Thesis, University of Newcastle Upon Tyne (1981).

[15] CHENEY, E. W., Introduction to Approximation Theory, McGraw-Hill, New York (1960).

[16] AHMED, A., Asymptotic properties of collocation projection norms, Vol. 19, No. 4, 45-50, 1990 Int. J. of Comp. and Math. with Applications. 


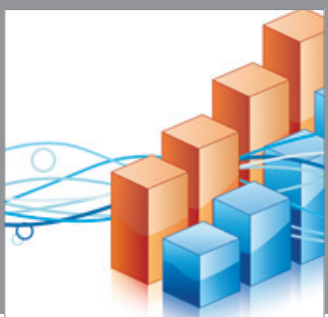

Advances in

Operations Research

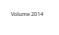

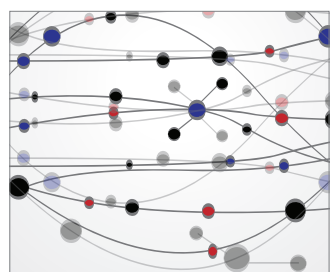

\section{The Scientific} World Journal
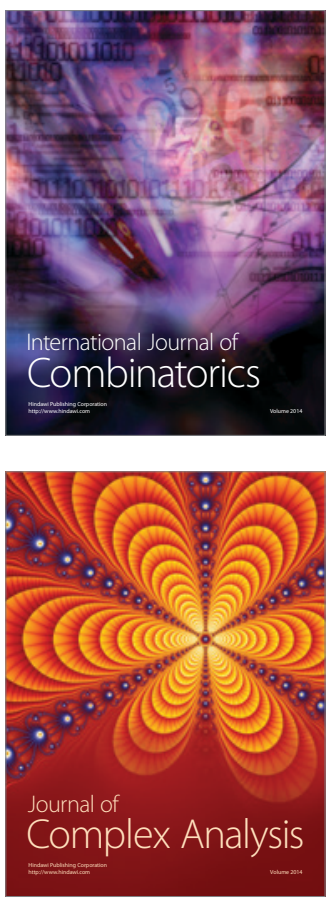

International Journal of

Mathematics and

Mathematical

Sciences
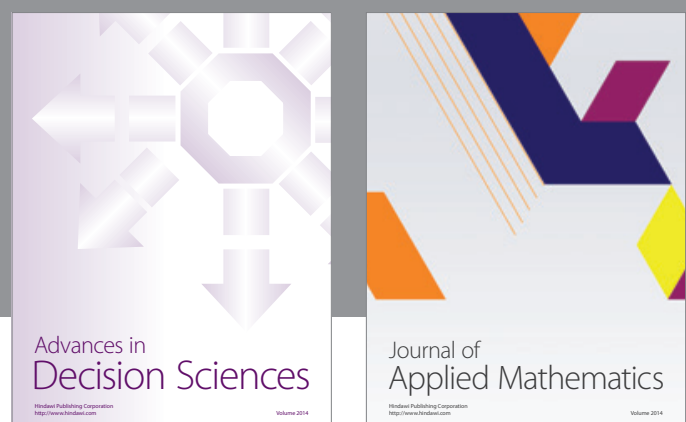

Journal of

Applied Mathematics
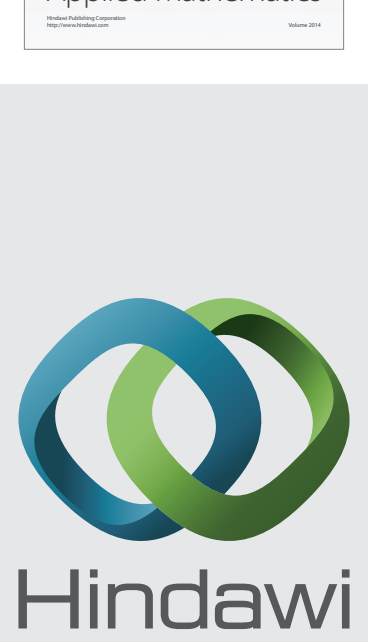

Submit your manuscripts at http://www.hindawi.com
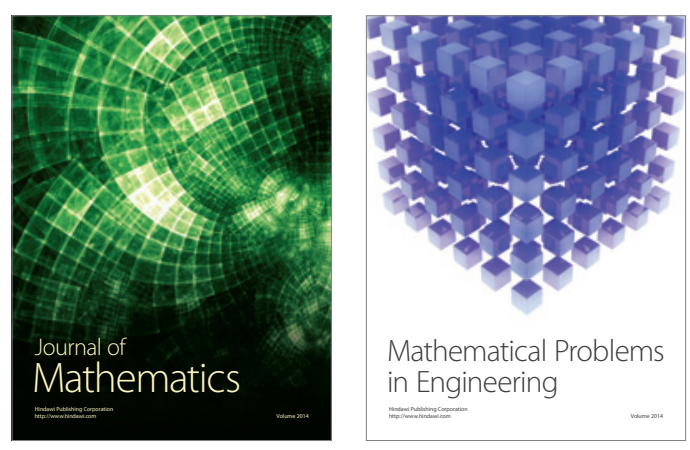

Mathematical Problems in Engineering
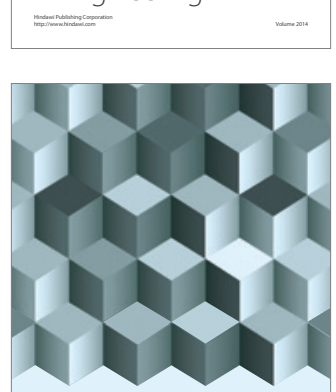

Journal of

Function Spaces
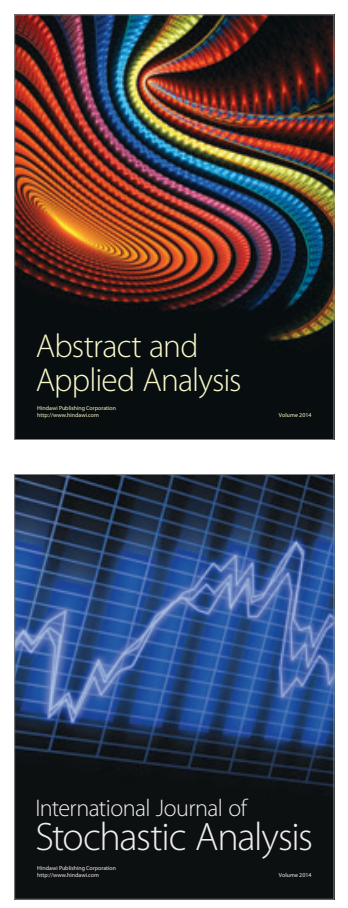

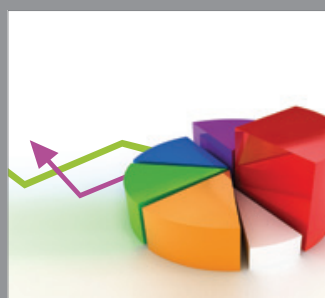

ournal of

Probability and Statistics

Promensencen
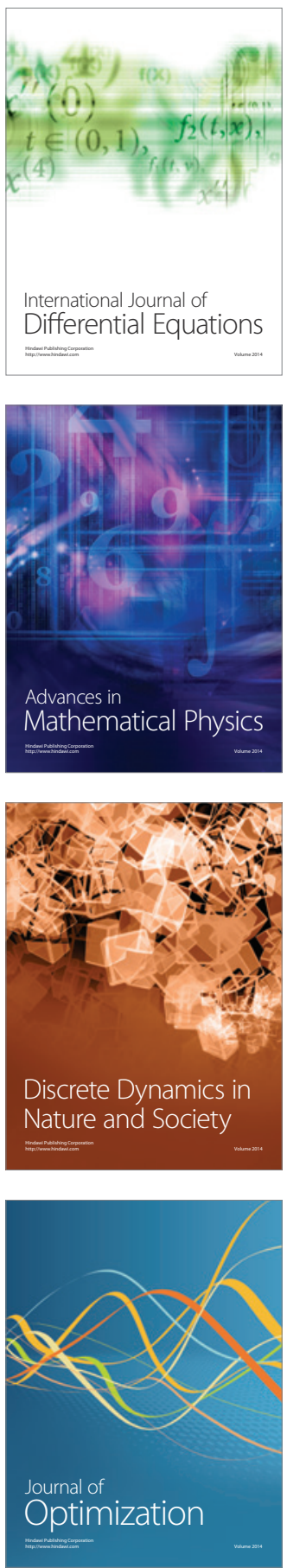